\title{
Intratumor heterogeneity of HMCN1 mutant alleles associated with poor prognosis in patients with breast cancer
}

\author{
Chie Kikutake ${ }^{1}$, Minako Yoshihara ${ }^{1,2}$, Tetsuya Sato ${ }^{1,2}$, Daisuke Saito ${ }^{1,2}$ and Mikita \\ Suyama ${ }^{1,2}$ \\ ${ }^{1}$ Medical Institute of Bioregulation, Kyushu University, Fukuoka 812-8582, Japan \\ ${ }^{2}$ AMED-CREST, Japan Agency for Medical Research and Development, Fukuoka 812-8582, Japan \\ Correspondence to: Mikita Suyama, email: mikita@bioreg.kyushu-u.ac.jp \\ Keywords: breast cancer; variant allele frequency; lymph node metastasis; genetic variant; next-generation sequencing \\ Received: November 21, $2017 \quad$ Accepted: August 15, $2018 \quad$ Published: September 07, 2018
}

Copyright: Kikutake et al. This is an open-access article distributed under the terms of the Creative Commons Attribution License 3.0 (CC BY 3.0), which permits unrestricted use, distribution, and reproduction in any medium, provided the original author and source are credited.

\section{ABSTRACT}

Human breast cancers comprise a complex and highly heterogeneous population of tumor cells. Intratumor heterogeneity is an underlying cause of resistance to effective therapies and disease recurrence. To explore prognostic factors based on intratumor heterogeneity, we analyzed genomic mutations in breast cancer patients registered in The Cancer Genome Atlas. We calculated the variant allele frequency (VAF) at each mutation site and evaluated the associations of VAFs with the prognosis of breast cancer. VAFs of HMCN1 correlated with the prognosis and lymph node status. Although the detailed function of HMCN1 remains unknown, it is located in extracellular matrix and the mutation in the gene might be associated with cancer cell invasion and metastasis. This finding suggests that HMCN1 is a potential metastatic factor and can be a candidate gene for targeted breast cancer therapy.

\section{INTRODUCTION}

Breast cancer is the most common type of cancer affecting women worldwide. In 2012, approximately 1.7 million cases of breast cancer were newly diagnosed [1]. Changes in dietary habits and a reduced birth rate can increase the risk of breast cancer. Breast cancer is a clinically heterogeneous disease for which four basic therapeutic or molecular subtypes have been classified based on the expression status of three receptors: estrogen receptor, progesterone receptor, and human epidermal growth factor receptor $2[2,3]$. Immunohistochemistry is used to classify these four tumor subtypes and ensure that effective treatment is provided to each patient.

Despite recent therapeutic advances, tumor recurrence and drug resistance remain major challenges in the field of breast cancer. These challenges are mainly attributed to intratumor heterogeneity [4], which is characterized by subclonal diversity within a tumor that originates from the accumulation of various somatic mutations during cell division and proliferation [5-7]. Intratumor heterogeneity has already been identified in several types of cancer, including breast, prostate, kidney, brain, liver, and lung cancers [8]. Drug-resistant subclones may develop via clonal evolution and reside at low frequencies within a tumor; after drug therapy, however, these subclones become the main population, leading to recurrence [9-11].

Intratumor heterogeneity can be most directly evaluated from DNA sequences using next-generation sequencing (NGS). One of the commonly used methods to analyze heterogeneity is the sequencing of samples from multiple regions of the same tumor [10, 12]. Ultradeep sequencing can also be used to detect mutations with extremely low allele frequencies. Variant allele frequency (VAF), calculated as the proportion of reads with mutations at the variant site, is used as an index of heterogeneity [13, 14]. VAFs in a tumor can be used to determine the cellular prevalence of a mutation within a sample and estimate subpopulation frequencies and the tumor evolutionary process [15-17]. For example, deep sequencing was used to evaluate mutational processes of 21 breast cancers, leading to the finding that every tumor harbored a distinct subclonal lineage [10]. The use of NGS and analytical methods to define clonal heterogeneity has also provided insights into the genetic processes underlying breast cancer metastasis [18]. Recent studies also showed that clonal distribution based on VAF correlated with prognosis 
$[19,20]$. Additionally, heterogeneity can be evaluated using large datasets generated by The Cancer Genome Atlas (TCGA) or the International Cancer Genome Consortium [21, 22]. Despite these advances, it remains highly challenging to identify tumor genetic factors associated with tumor growth or metastasis, as tumors exhibit considerable heterogeneity.

Several recent studies based on TCGA data have focused on the identification of driver genes and identified pathways containing potential drug targets [23]. These studies have accelerated the development of pathwayspecific inhibitory drugs. Although mutations in breast cancer driver genes such as TP53, PIK3CA, and GATA3 have been extensively investigated, somatic alterations in other genes are also believed to be associated with breast cancer [24]. To gain better insights into the extent of intratumor heterogeneity, we analyzed breast cancer genome sequencing data from TCGA. In this study, we focused on genes associated with breast cancer prognosis.

\section{RESULTS}

\section{Identification of genes with high frequencies of mutations}

We sought genes with one of four types of mutation (missense mutations, nonsense mutations, frameshift insertions, and frameshift deletions) in $\geq 50$ samples derived from the 1,044 breast cancer datasets in TCGA. We identified 17 such genes (Table 1) and calculated the mean VAF for each in the sample containing mutations (Figure 1). All VAFs were adjusted for tumor purity taken from the previous study [25]. The mean VAFs of already known driver genes in breast cancer, such as TP53, $P I K 3 C A$, and $C D H 1$ were found to be relatively high.

To examine the association of overall survival (OS) with VAFs of these 17 genes, we applied a Cox proportional hazards regression analysis with the covariates of age, tumor grade, and VAFs. In this analysis, the samples were divided into two groups using a VAF of 0.30 (i.e., $30 \%$ ) as a cutoff. We used this cutoff because a previous study, which focused on samples with high tumor purity ( $\geq 70 \%$ ), considered that a VAF of $\geq 0.25$ was more likely to be clonal, whereas lower values were more likely to be subclonal [20]. Assuming that average purity is $85 \%$ (range $70-100 \%$ ) then the cutoff should be $0.3(0.25 / 0.85$ $=0.294)$. We conducted this analysis without adjusting for other covariates just for a screening of genes that are possibly associated with breast cancer prognosis. We corrected $P$ values for multiple testing using Benjamini and Hochberg false discovery rate (FDR) [26]. VAFs of $H M C N 1$ was found to be possibly associated with breast cancer prognosis (FDR $<0.1)$ (Table 1$)$, and we focused on $H M C N 1$, for which no association with breast cancer has previously been reported.

A total of 78 somatic mutations in $H M C N 1$ were detected in $6.1 \%(64 / 1,044)$ of samples (Table 2 and Figure 2). Among samples with detectable HMCN1 mutations, 9.4\% (6/64) contained two distinct mutations and $3.1 \%(2 / 64)$ contained more than two mutations. Of the $78 H M C N 1$ mutations, $82.1 \%(64 / 78)$ were missense, whereas $10.3 \%(8 / 78)$ were nonsense and $7.7 \%(6 / 78)$ were indels. Furthermore, 69.2\% (54/78) of the mutations were clustered in the Ig-like C2-type domains of HMCN1.

To evaluate the existence of any association between the mutation type and VAFs, we applied a one-

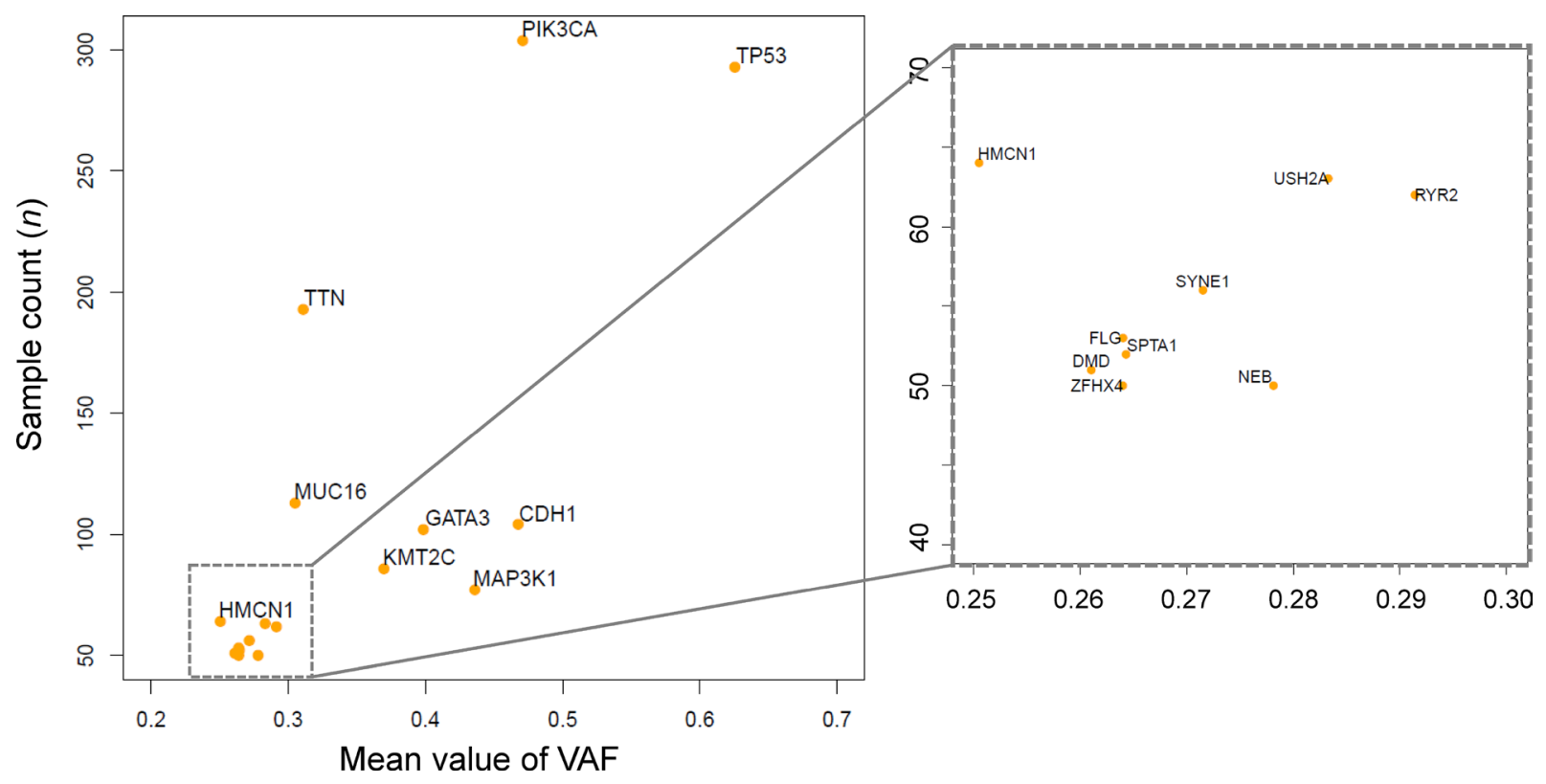

Figure 1: Frequently mutated genes and mean variant allele frequencies (VAFs). The scatter plot depicts 17 genes harboring mutations in $>50$ samples. The $\mathrm{x}$-axis indicates the mean VAF, and the $\mathrm{y}$-axis indicates the number of samples with mutations. The plot at right is an enlargement of the square area enclosed by dotted lines in the left plot. 
Table 1: Frequently mutated genes and mean variant allele frequencies and hazard ratios

\begin{tabular}{cccccc}
\hline Gene & Sample count & Mean value of VAF & Hazard Ratio (95\% CI) & $\boldsymbol{P}$-value & FDR $^{\mathbf{a}}$ \\
\hline PIK3CA & 304 & 0.471 & $1.78(0.729-4.348)$ & 0.206 & 0.696 \\
TP53 & 293 & 0.626 & $1.276(0.455-3.581)$ & 0.643 & 0.994 \\
$T T N$ & 193 & 0.311 & $1.85(0.843-4.06)$ & 0.125 & 0.531 \\
MUC16 & 113 & 0.305 & $1.768(0.619-5.048)$ & 0.287 & 0.696 \\
CDH1 & 104 & 0.468 & $1.019(0.263-3.951)$ & 0.979 & 0.999 \\
GATA3 & 102 & 0.398 & $0.859(0.245-3.01)$ & 0.813 & 0.999 \\
KMT2C & 86 & 0.370 & $1.327(0.478-3.687)$ & 0.587 & 0.994 \\
MAP3K1 & 77 & 0.436 & $0.114(0.013-0.985)$ & 0.048 & 0.287 \\
HMCN1 & 64 & 0.251 & $11.441(2.065-63.406)$ & 0.005 & $0.090^{*}$ \\
USH2A & 63 & 0.283 & $1.185(0.245-5.74)$ & 0.833 & 0.999 \\
RYR2 & 62 & 0.291 & $0.059(0.003-1.008)$ & 0.051 & 0.287 \\
SYNE1 & 56 & 0.272 & $1.635(0.11-24.181)$ & 0.721 & 0.999 \\
$F L G$ & 53 & 0.264 & $0.342(0.04-2.923)$ & 0.327 & 0.696 \\
SPTA1 & 52 & 0.264 & $1.965(0.531-7.274)$ & 0.312 & 0.696 \\
DMD & 51 & 0.261 & $1.87(0.396-8.831)$ & 0.429 & 0.811 \\
NEB & 50 & 0.278 & $1.124(0.135-9.342)$ & 0.914 & 0.999 \\
ZFHX4 & 50 & 0.264 & & - & - \\
\hline
\end{tabular}

Abbreviations: 95\% CI, 95\% confidence interval; VAF, variant allele frequency; FDR, false discovery rate.

${ }^{a}$ Astarisk indicates FDR $<0.1$.

${ }^{\mathrm{b}} \mathrm{ZFHX} 4 \mathrm{VAF}$ could not be analyzed because sample size is small.

way ANOVA to the data and found that the mean VAF values did not significantly differ among the four types of mutations $(P=0.430)$ (Supplementary Figure 1). We further evaluated the associations of the four molecular breast cancer subtypes with VAFs of $H M C N 1$. However, an ANOVA indicated that the mean VAF values did not significantly differ among the four subtypes $(P=0.060)$ (Supplementary Figure 2).

\section{Expression of HMCN1}

We compared the relative HMCN1 mRNA expression levels among samples with higher (VAF of $\geq 0.30, n=19$ ) and lower VAFs (VAF of $<0.30, n=45$ ).
As a result, we found that $H M C N 1$ expression levels did not significantly differ between the two groups $(P=0.343)$ (Supplementary Figure 3A). Additionally, we compared TP53 and PIK3CA expression levels between the VAF groups and found no significant differences in either $(P=0.515$ and 0.300 , respectively) (Supplementary Figure $3 \mathrm{~B}$ and $3 \mathrm{C}$ ). We also found no significant differences in the relative $H M C N 1$ mRNA expression levels between $H M C N 1$ mutant and wild-type samples $(P=0.984)$ (Supplementary Figure 3D).

To identify genes for which the expression levels were associated with the $H M C N 1 \mathrm{VAF}$, we analyzed mRNA expression levels of all annotated genes. Among the annotated genes, only two significantly exhibited

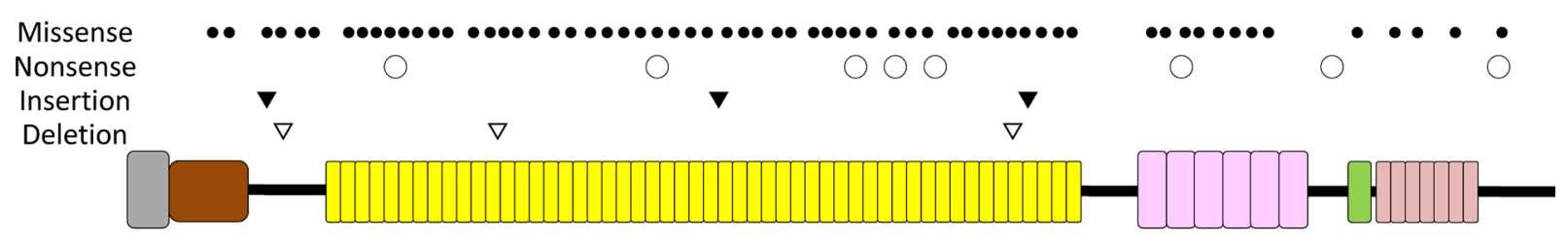

Signal Peptide

von Willebrand A domain

Ig-like C2-type domains
TSP type-1 domains

G2 Nidogen domain

EGF-like domains

Figure 2: A schematic of the domains of human HMCN1 (hemicentin-1). The types and positions of 78 somatic mutations are indicated above the diagram. 
Table 2: Distribution of HMCN1 mutations

\begin{tabular}{|c|c|c|c|c|c|}
\hline HMCN1 Domains & $\begin{array}{l}\text { Missense } \\
\text { mutation }\end{array}$ & $\begin{array}{l}\text { Nonsense } \\
\text { mutation }\end{array}$ & Deletion & Insertion & Total \\
\hline VWFA domain & 2 & 0 & 0 & 0 & 2 \\
\hline Ig-like C2-type domains & 45 & 5 & 2 & 2 & 54 \\
\hline TSP type-1 domains & 8 & 1 & 0 & 0 & 9 \\
\hline Nidogen $\mathrm{G} 2$ beta-barrel domain & 1 & 1 & 0 & 0 & 2 \\
\hline EGF-like domains & 3 & 0 & 0 & 0 & 3 \\
\hline Other & 5 & 1 & 1 & 1 & 8 \\
\hline Total & 64 & 8 & 3 & 3 & 78 \\
\hline
\end{tabular}

Abbreviations: EGF, epidermal growth factor; Ig, immunoglobulin; TSP, thrombospondins; VWFA, von Willebrand factor type A.

different expression in terms of the $H M C N 1$ VAF. A high $C A 9$ and $C A S P 14$ expression level $(P=0.043$ and 0.024 , respectively) and low MTRNR $2 L 1$ and TCN1 expression level ( $P=0.024$ and 0.043 , respectively) were found to be significantly associated with a higher VAF (Figure 3). CA9 encodes carbonic anhydrase IX, an endogenous marker of hypoxic cells in breast cancers. CASP14 encodes caspase14, which is one of the apoptosis-related cysteine peptidase. MTRNR2L1 encodes human MT-RNR2-like 1, for which detailed functions remain unknown and TCN1 encodes a member of the vitamin B12-binding protein family, named "transcobalamin 1".

\section{Relationship between intratumor heterogeneity and $H M C N 1$ VAFs}

To investigate intratumor heterogeneity in individuals with $H M C N 1$ mutations, we measured the
A

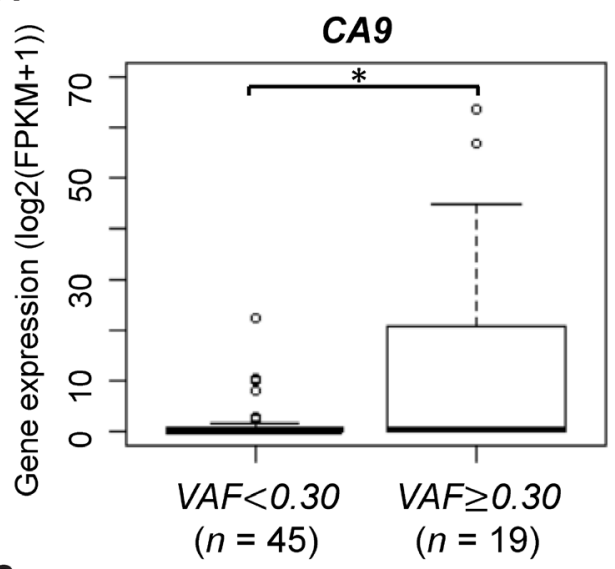

C

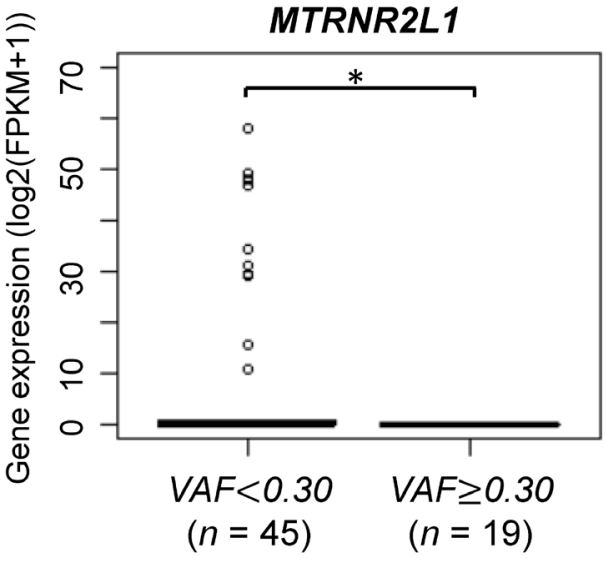

B

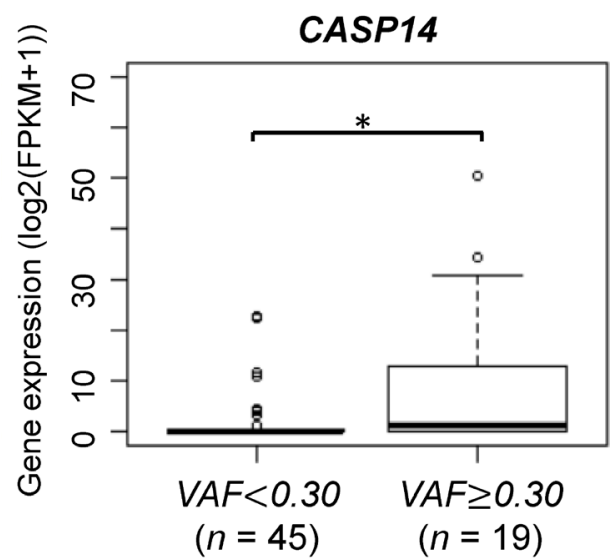

D

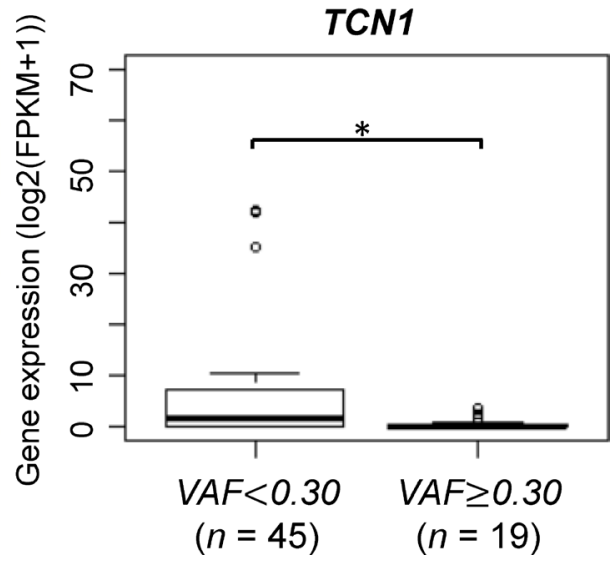

Figure 3: $C A 9$ and $M T R N R 2 L 1$ mRNA expression according to $H M C N 1$ variant allele frequencies (VAFs). Samples were divided into two groups using a VAF cutoff of $0.30(<0.30, n=45$ and $\geq 0.30, n=19)$. The asterisk indicates statistical significance. 
number of subclones across each sample. We performed this analysis using the SciClone [16] with VAFs from somatic SNVs and copy number estimates. We compared between the higher and the lower HMCN1 VAF groups in terms of the number of subclones by Fisher's exact test. The distributions of the number of subclones were not significantly different between the two groups $(P=0.347)$ (Supplementary Figure 4A).

There is another index for intratumor heterogeneity, mutant-allele tumor heterogeneity (MATH), which can be calculated from VAF distribution in a sample [27]. Previous studies have shown that higher MATH score was correlated with poor prognosis in head and neck squamous cell carcinoma and colon cancer $[27,28]$. We compared between the higher and the lower HMCN1 VAF groups in terms of MATH scores by Wilcoxon signed rank test. In the 19 samples with higher HMCN1 VAF, the mean MATH was $35.014(S D=11.300)$. Meanwhile, in the 45 samples with lower HMCN1 VAF, the mean MATH was $33.919(S D=10.557)$. No significant differences between the two groups were detected $(P=0.771)$ (Supplementary Figure 4B). These findings indicate that the prevalence of the mutations in HMCN1 might not be involved in the status of intratumor heterogeneity.

\section{Associations of $H M C N 1$ with common driver genes}

As TP53 and PIK3CA mutations are among the most common genetic aberrations in breast cancers [23], we compared the VAFs of these two driver genes with those of HMCN1. Among the 64 samples harboring mutations in HMCN1, 22 and 23 also harbored mutations in TP53 and $P I K 3 C A$, respectively, and five harbored mutations in both genes.

In the 22 samples with both TP53 and HMCN1 mutations, the mean TP53 VAF was $0.697(S D=0.249)$ and the mean HMCN1 VAF was $0.288(S D=0.201)$. Meanwhile, in the 23 samples with both $P I K 3 C A$ and HMCN1 mutations, the mean PIK3CA VAF was 0.442 $(S D=0.269)$ and the mean HMCN1 VAF was 0.230 $(S D=0.148)$. A paired $t$-test showed that VAFs of the two driver genes were significantly higher than that of HMCN1 (TP53; $P<0.01$, PIK3CA; $P<0.01$ ) (Supplementary Figure 5), indicating that mutations in HMCN1 occurred later in the tumor evolutionary process than the mutations in TP53 and PIK3CA. This finding suggests that the mutations in HMCN1 might be involved in breast cancer progression.

\section{HMCN1 mutations and clinical outcomes}

Next, we evaluated the association between $H M C N 1$ mutation status and clinical variables by $\chi^{2}$ test or Fisher's exact test. We found tumor size $(P=0.028)$ and molecular subtype $(P=0.021)$ were related with the HMCN1 mutation (Table 3). To assess the relationship of the $H M C N 1$ VAF with prognosis, the 64 samples harboring
HMCN1 mutations were divided into two groups according to VAFs and subjected to an OS analysis. These groups were also compared with individuals without $H M C N 1$ mutations (wild-type; WT). The resulting Kaplan-Meier plot shows that a higher HMCN1 VAF significantly correlated with poor prognosis (log-rank test: vs. WT; $P=0.022$ and vs. VAF of $<0.30 ; P=0.015$ ) (Figure 4 ). Concordantly, in a multivariate Cox proportional hazards regression analysis adjusted for the covariates of lymph node status, tumor grade, tumor size, and age, the VAF $(P=0.036)$ and lymph node status $(P=0.012)$ were significantly associated with poor prognosis (Table 4$)$.

To exclude the possibility that this significant association is not attributable to bias in the impact of mutations in the two groups, we evaluated the impact of single nucleotide variants in $H M C N 1$ on protein structure and function using PolyPhen-2 scores [29]. The Pearson correlation coefficient between VAFs and PolyPhen-2 scores of HMCN1 was $-0.204(P=0.151)$, indicating no significant correlation. To analyze the relationship between prognosis and PolyPhen-2 scores, the 64 samples were divided into two groups using a PolyPhen- 2 score of 0.85 as a cutoff (higher, $n=27$ and lower, $n=24$ ); this score ranges from 0 to 1 and yields predictions of "probably damaging" $(>0.85)$, "possibly damaging" (0.85-0.15), or "benign" $(<0.15)$. We found that the Polyphen-2 score of nonsynonymous HMCN1 mutations did not significantly associate with breast cancer prognosis (PolyPhen-2 scores of $<0.85$ vs. WT; $P=0.801$ and PolyPhen-2 scores of $\geq 0.85$ vs WT; $P=$ 0.671) (Supplementary Figure 6).

These results suggest that the HMCN1 VAF is an independent prognostic factor for OS, such that a higher VAF may be associated with poor survival in patients with breast cancer.

\section{Correlations with potential prognostic factors}

We next evaluated the association of the HMCN1 VAF with individual clinical characteristics (lymph node status, tumor grade, tumor size, and age) in the 64 tumor samples, which were divided into three groups by lymph node status: N0, N1, and N2-N3. Samples were also divided into three groups by tumor grade: grades 1,2, and 3-4. Regarding lymph node status, tumor grade, and tumor size, we examined whether a higher VAF was associated with significantly higher stages of clinical features using the Cochran-Armitage trend test. We found a significant association of a higher VAF with a much higher lymph node status $(P=0.029)$ (Figure 5A). By contrast, the tumor grade $(P=0.151)$ and tumor size $(P=0.283)$ were not significantly associated with the HMCN1 VAF (Figure $5 \mathrm{~B}$ and $5 \mathrm{C})$. The mean ages of patients $(n=64)$ in the higher and lower VAF groups were $58.05(S D=17.95)$ years and $61.41(S D=11.85)$ years, respectively. A $t$-test revealed no significant difference in the mean age between the groups $(P=0.461)$ (Figure 5D). 
Table 3: Clinical data of 1,044 breast cancer patients

\begin{tabular}{|c|c|c|c|c|c|}
\hline \multirow[b]{3}{*}{ Variables } & & Overall & WT & MT & \multirow[b]{3}{*}{$P$-value } \\
\hline & & $n=1,044$ & $n=980$ & $n=64$ & \\
\hline & & No. $(\%)$ & No. $(\%)$ & No. $(\%)$ & \\
\hline \multirow[t]{4}{*}{ Lymh node status } & & & & & 0.175 \\
\hline & Negative & $485(46.5)$ & $450(45.9)$ & $35(54.7)$ & \\
\hline & Positive & $540(51.7)$ & $513(52.3)$ & $27(42.2)$ & \\
\hline & Unknown & $19(1.8)$ & $17(1.7)$ & $2(3.1)$ & \\
\hline \multirow[t]{6}{*}{ Tumor grade } & & & & & 0.080 \\
\hline & 1 & $172(16.5)$ & $163(16.6)$ & $9(14.1)$ & \\
\hline & 2 & $582(55.7)$ & $536(54.7)$ & $46(71.9)$ & \\
\hline & 3 & $239(22.9)$ & $231(23.6)$ & $8(12.5)$ & \\
\hline & 4 & $20(1.9)$ & $19(1.9)$ & $1(1.6)$ & \\
\hline & Unknown & $31(3.0)$ & $31(3.2)$ & 0 & \\
\hline \multirow[t]{5}{*}{ Tumor size $(\mathrm{cm})$} & & & & & $0.028^{*}$ \\
\hline & $<2$ & $267(25.6)$ & $255(26.0)$ & $12(18.8)$ & \\
\hline & $2-5$ & $603(57.8)$ & $556(56.7)$ & $47(73.4)$ & \\
\hline & $\geqq 5$ & $171(16.4)$ & $166(16.9)$ & $5(7.8)$ & \\
\hline & Unknown & $3(0.3)$ & $3(0.3)$ & 0 & \\
\hline \multirow[t]{7}{*}{ Molecular subtype } & & & & & $0.021^{*}$ \\
\hline & Luminal A & $401(38.4)$ & $385(39.3)$ & $16(25.0)$ & \\
\hline & Luminal B & $171(16.4)$ & $163(16.6)$ & $8(12.5)$ & \\
\hline & HER2-enriched & $65(6.2)$ & $58(5.9)$ & $7(10.9)$ & \\
\hline & Basal-like & $132(12.6)$ & $123(12.6)$ & $9(14.1)$ & \\
\hline & Normal & $23(2.2)$ & $19(1.9)$ & $4(6.3)$ & \\
\hline & Unknown & $252(24.1)$ & $232(23.7)$ & $20(31.3)$ & \\
\hline \multirow[t]{5}{*}{ Age (year) } & & & & & 0.450 \\
\hline & Median (range) & $59(27-90)$ & $59(27-90)$ & $59(34-90)$ & \\
\hline & $<50$ & $276(26.4)$ & $261(26.6)$ & $15(23.4)$ & \\
\hline & $\geqq 50$ & $743(71.2)$ & $649(66.2)$ & 49 (76.6) & \\
\hline & Unknown & $25(2.4)$ & $25(2.6)$ & $0(0)$ & \\
\hline
\end{tabular}

Abbreviations: 95\% CI, 95\% confidence interval; MT; HMCN1 mutant, VAF; variant allele frequency; WT, wild-type. ${ }^{\mathrm{a}}$ Astarisk indicates statistical significance.

We also used other 15 types of cancer dataset from TCGA and examined the association between HMCN1 VAFs and OS. In the 15 types of cancer, only cervical squamous cell carcinoma and endocervical adenocarcinoma (CESC) samples showed significantly poorer OS in the samples with higher $H M C N 1$ VAFs $(n=7, \mathrm{VAF} \geq 0.30)$ than those with lower VAFs $(n=15$, VAF < 0.30) (log-rank test: $P=0.048)$ (Supplementary Figure 7). Although, we applied a Cox proportional hazards regression analysis with the covariates of age, tumor grade, and HMCN1 VAFs, the VAFs were not associated with poor prognosis $(\mathrm{HR}=5.436,95 \% \mathrm{CI}$ : $0.543-54.432, P=0.150)$.

\section{DISCUSSION}

Breast cancers are known to exhibit a large degree of genetic heterogeneity. In this study, we analyzed intratumor heterogeneity using VAFs calculated from a set of breast cancer cases registered in TCGA. Through a VAF-based analysis of mutations, we showed that VAFs of $H M C N 1$ was possibly associated with breast cancer prognosis. Although the detailed function of $H M C N 1$ in humans remains unknown, mutations in $H M C N 1$ might be associated with cancer cell invasion and metastasis. Using the data of the DRIVE dataset, the CIMBA dataset, and the Foundation One dataset from breast cancer patients, 
Table 4: Multivariate Cox proportional hazards regression analysis of overall survival according to the clinical characteristics of 64 breast cancer patients

\begin{tabular}{|c|c|c|}
\hline Variables & Hazard Ratio (95\% CI) & $P$-value ${ }^{a}$ \\
\hline \multicolumn{3}{|l|}{ Lymph node status } \\
\hline Positive vs Negative & $97.931(2.709-3539.805)$ & $0.012^{*}$ \\
\hline \multicolumn{3}{|l|}{ Tumor grade } \\
\hline $3-4$ vs $1-2$ & $9.468(0.042-2147.487)$ & 0.417 \\
\hline \multicolumn{3}{|l|}{ Tumor size, cm } \\
\hline $2-5 \mathrm{vs} \leq 2$ & $1.281(0.159-10.302)$ & 0.816 \\
\hline$>5$ vs $\leq 2$ & $29.032(0.284-2966.692)$ & 0.154 \\
\hline \multicolumn{3}{|l|}{ Age } \\
\hline$\geq 50$ vs $<50$ & $0.114(0.011-1.169)$ & 0.068 \\
\hline \multicolumn{3}{|l|}{ VAF } \\
\hline$\geq 0.30$ vs $<0.30$ & $17.950(1.216-264.976)$ & $0.036^{*}$ \\
\hline
\end{tabular}

Abbreviations: 95\% CI, 95\% confidence interval; VAF, variant allele frequency.

${ }^{a}$ Astarisk indicates statistical significance.

we could not validate our result because the $H M C N 1$ VAF and survival time information could not be obtained. In cervical cancer from TCGA, however, survival time between the two groups of HMCN1 VAF values were also significantly different. Cervical cancer, like breast cancer, is known to associated with hormone estrogen. Therefore, this result may support the prognostic impact of HMCN1 on breast cancer. It will be possible to further evaluate the validity of our results by accumulating more cohorts.

HMCN1 encodes a large extracellular protein belonging to the immunoglobulin superfamily and comprises several distinct domains, including the von Willebrand factor and Ig-like C2-type domains [30]. HMCN1 mutations are believed to correlate with agerelated macular degeneration [31]. According to another recent report, HMCN1 acts as a suppressor of gallbladder cancer metastasis [32] and is commonly mutated in certain samples of head and neck squamous cell carcinoma [33]. There are at least three possible functional implications of the mutations in HMCN1 in breast cancer metastasis. First, $H M C N 1$ is also known as FBLN6 (fibulin 6) and one of the extra cellular matrix (ECM) proteins $[34,35]$. The

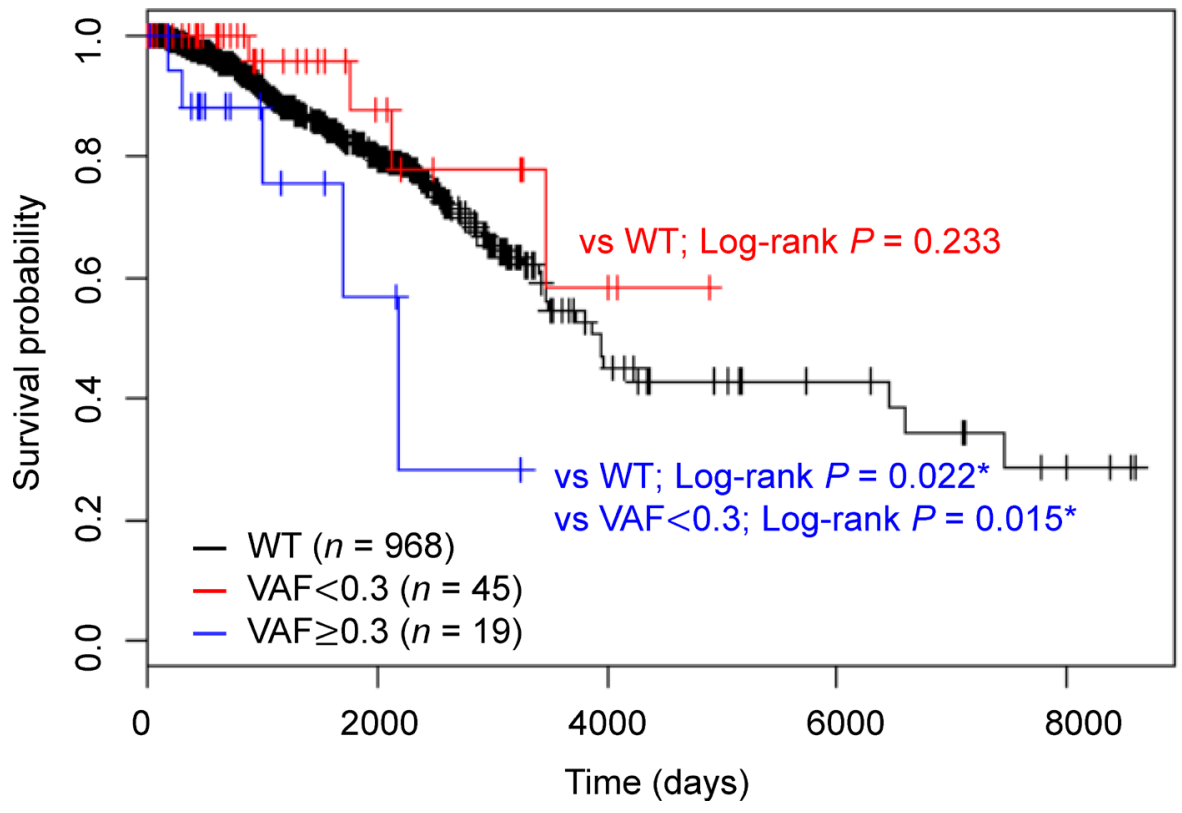

Figure 4: Kaplan-Meier analysis of overall survival according to HMCN1 variant allele frequencies (VAFs). Samples were divided into three groups using a VAF cutoff of $0.30(<0.30$, red, $n=45$ and $\geq 0.30$, blue, $n=19)$ or WT (black, $n=968)$. The log-rank test was used to evaluate the statistical significance of the difference between the two survival curves (VAF of $\geq 0.30$ vs. VAF of $<0.30$, $\mathrm{VAF}$ of $<0.30 \mathrm{vs}$. WT and VAF of $\geq 0.30$ vs WT). 
fibulins are shown to be involved in basement membrane and formation of stable cell-to-cell interactions, leading to organization and stabilization to ECM structure [36]. When HMCN1 does not function properly in cancer cell, sufficient cell adhesion might be inhibited and as a result of promoting cancer invasion due to instability of HMCN1 caused by the variants in the gene. For example, previous study reported that epigenetically silenced fibulin 5 promotes invasion and metastasis in lung cancer [37]. Second, HMCN1, which contains estrogen receptor binding site, seems to be associated with postpartum depression symptoms [38], and one of its functions is suggested to be cell adhesion [39]. Therefore, HMCN1 mutations may be associated to cancer proliferation and metastasis because of the disruption of these functions. Finally, other studies have shown that HMCN1 might interact with DDX1 [40], a DEAD box protein with RNA helicase activity [41, 42]. Notably, the expression of $D D X 1$ was reported to decrease under hypoxic conditions [43], and intratumor hypoxia is associated with cancer metastasis and, consequently, patient mortality [44, 45]. Indeed, an earlier report found that $D D X 1$ correlated with ovarian tumor metastasis and progression [46]. In

A

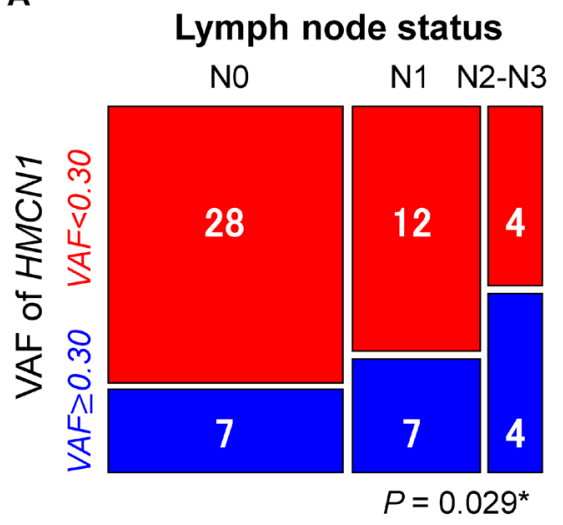

C

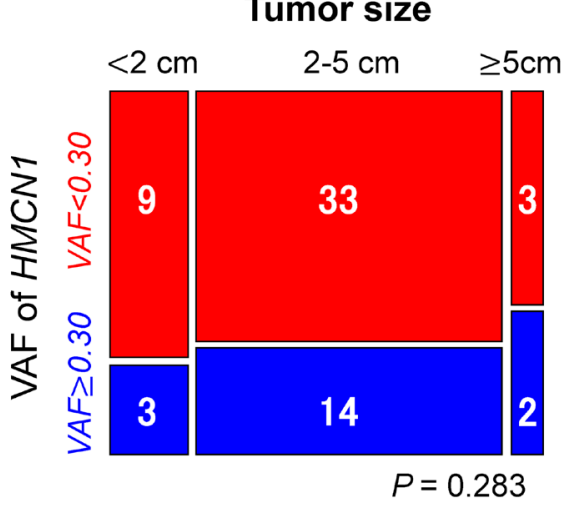

the current study, we found that $C A 9$, the expression of which is also associated with tumor hypoxia [43, 47], was expressed at significantly higher levels in patients with higher HMCN1 VAFs than in those with lower VAFs. Therefore, the HMCN1 VAF may indicate the metastatic potential of a breast cancer.

Although metastasis is the main cause of death among breast cancer patients, factors involved in metastasis remain poorly characterized. It is more difficult to identify genetic factors associated with metastasis, a complex process, than to identify driver genes [48]. Differences in genetic heterogeneity between metastatic and primary tumors may affect treatment efficacy and thus represent one of the biggest obstacles toward cure for breast cancer. Using VAFs to explicitly address heterogeneity, we successfully identified $H M C N 1$ as a possible metastatic factor. Although further experimental validation is needed to determine the involvement of HMCN1 in metastasis, this approach could be used to screen genes that have not previously been investigated.

In this study, we focused on nonsynonymous mutations and indels. However, intratumor heterogeneity may also be caused by copy number variants or mutations

B

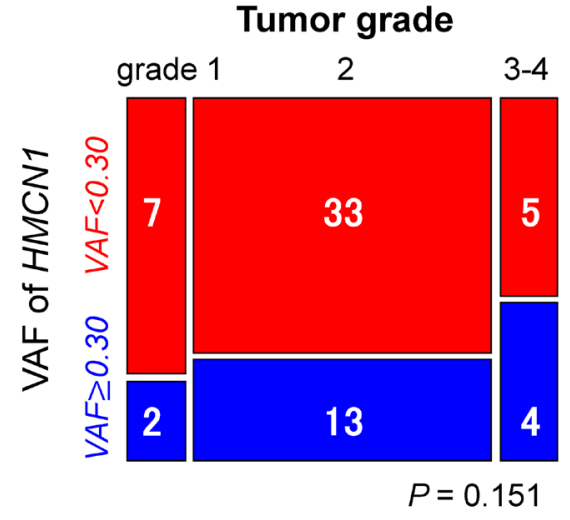

D

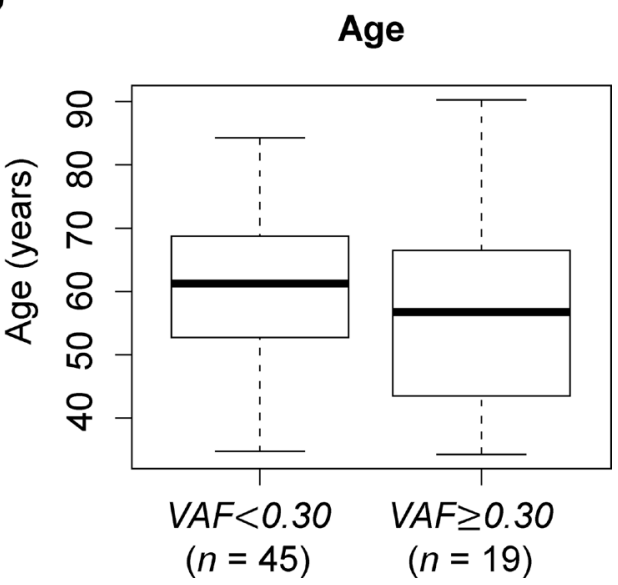

Figure 5: Associations of the $H M C N 1$ variant allele frequency (VAF) with clinical characteristics of (A) lymph node status, (B) tumor grade, $(\mathbf{C})$ tumor size, and (D) patient age. Samples were divided into two groups using a VAF cutoff of $0.30(<0.30$, red, $n=45$ and $\geq 0.30$, blue, $n=19$ ). Blue and red squares in mosaic plots indicate sample counts from the higher and lower VAF groups, respectively. In the TCGA dataset, the lymph node status for two cases were not provided. The asterisk indicates statistical significance. 
in noncoding regions, such as those in cis-regulatory elements and splice sites [49-52]. Moreover, epigenetic alterations can also promote cancer progression [53]. As genome-wide epigenetic datasets from normal cells grow rapidly [54], epigenome data analyses of cancer cells will allow evaluations of the impacts of epigenetic factors on intratumor heterogeneity.

In conclusion, to our knowledge, this is the first study to identify $H M C N 1$ as a potential metastatic factor in breast cancer using a comparative analysis of genomic and transcriptomic data registered in TCGA. In addition to the standard classification of breast tumors based on the four molecular types, the use of VAFs, which reflect tumor evolution, might provide further genetic profile information that can be used to characterize tumor samples. Our approach allows us to identify new diagnostic markers or candidate genes for targeted therapy and is therefore expected to facilitate precision medicine.

\section{MATERIALS AND METHODS}

\section{Datasets}

A total of 1,080 RNA-seq and variant datasets from breast cancers were downloaded from TCGA (https:// portal.gdc.cancer.gov/). For variant data, we used VCF files generated by comparing matched tumor-normal pairs using the Mutect2 software package. We also downloaded the associated clinical patient data. Similarly, dataset from other 15 types of cancer were obtained from TCGA: Bladder urothelial carcinoma (BLCA; $n=416$ ), cervical squamous cell carcinoma and endocervical adenocarcinoma (CESC; $n=307)$, colon adenocarcinoma (COAD; $n=$ $605)$, glioblastoma multiforme (GBM; $n=938)$, head and neck squamous cell carcinoma (HNSC; $n=512$ ), kidney renal clear cell carcinoma (KIRC; $n=697$ ), lower grade glioma (LGG; $n=938$ ), liver hepatocellular carcinoma (LIHC; $n=378$ ), lung adenocarcinoma (LUAD; $n=$ 587), lung squamous cell carcinoma (LUSC; $n=503$ ), ovarian serous cystadenocarcinoma (OV; $n=443)$, prostate adenocarcinoma (PRAD; $n=503$ ), skin cutaneous melanoma (SKCM; $n=472$ ), thyroid carcinoma (THCA; $n$ $=504$ ), and uterine corpus endometrial carcinoma (UCEC; $n=604)$.

\section{Mutation analysis}

In this study, we only considered mutations with a coverage depth of $\geq 20$. We extracted gene mutations [i.e., nonsynonymous substitutions (missense and nonsense mutations) and indels (frameshift insertions and frameshift deletions)] observed in $\geq 50$ samples. We used this cutoff because the lower limit of the average mutation rate for significantly mutated genes was approximately 2-4\% [23]. VAFs were calculated as the proportion of variant allele reads to total reads at the mutation site. When a sample harbored multiple mutations in the same gene, the larger VAF was used as the VAF for the gene. The VAF was adjusted for tumor purity estimate. This estimate, which was derived from immunohistochemistry analysis, was downloaded from the previous study [25].

The number of subclones in a tumor cell were inferred by both VCF files and DNA copy number variation data using the R package SciClone with default settings [16].

\section{Statistical analysis}

Statistical analysis was conducted using the $\mathrm{R}$ software, version 3.3.1 (R Project for Statistical Computing, Vienna, Austria), and JMP Pro, version 13.0 (SAS Institute Inc., Cary, NC, USA). A $\chi^{2}$ test or Fisher's exact test (when $\geq 1$ cells had an expected frequency of $\leq 5$ in any clinical group) was used to evaluate the relationships between the mutation status and clinical variables. We also used the test for comparison the number of subclones. OS was estimated using the Kaplan-Meier method in the R survival package (version 2.41-3). For the multivariate analysis, adjusted hazard ratios (HRs) with 95\% confidence intervals $(95 \%$ CIs) were calculated using a Cox proportional hazards regression model. We used edgeR (version 3.16.5), a Bioconductor package (http://www.bioconductor.org/ packages/release/bioc/html/edgeR.html), to detect genes differentially expressed between two groups. For each gene, the $\mathrm{R}$ exactRankTests package (version 0.8-28) was used to evaluate the difference in both expression levels and MATH between groups of samples. For categorical data such as tumor grade, tumor size category, and lymph node status, we used a one-sided Cochran-Armitage trend test to evaluate the existence of a linear relationship in terms of VAFs. We used Welch's $t$-test to compare continuous data between the two groups. An analysis of variance (ANOVA) model was used to compare the mean values of more than two groups. $P$-values were considered statistically significant at $<0.05\left({ }^{*} P<0.05,{ }^{* *} P<0.01\right)$.

\section{Author contributions}

Chie Kikutake: Conception and design of study, writing original draft, and data analysis. Minako Yoshihara: Data interpretation, review, and supervision. Tetsuya Sato: Data interpretation, review, and supervision. Daisuke Saito: Data interpretation, review, and supervision. Mikita Suyama: Data review and interpretation, writing review and editing final draft, supervision, and project administration. All authors reviewed and approved the article.

\section{ACKNOWLEDGMENTS}

This work was supported by the "Advanced Computational Scientific Program" of the Research Institute for Information Technology, Kyushu University. The results shown here are partly based 
upon data generated by the TCGA Research Network: http://cancergenome.nih.gov/.

\section{CONFLICTS OF INTEREST}

The authors declare they have no competing interests.

\section{FUNDING}

This study was supported by JSPS Grants-in-Aid for Scientific Research [Grant number: 17H03619 to M.S. and 17KT0128, 17K07257 to T.S.] and The Shin-Nihon of Advanced Medical Research [to T.S.].

\section{REFERENCES}

1. Torre LA, Bray F, Siegel RL, Ferlay J, Lortet-tieulent J, Jemal A. Global Cancer Statistics, 2012. CA Cancer J Clin. 2015; 65:87-108.

2. Perou CM, Sørlie T, Eisen MB, van de Rijn M, Jeffrey SS, Rees CA, Pollack JR, Ross DT, Johnsen H, Akslen LA, Fluge O, Pergamenschikov A, Williams C, et al. Molecular portraits of human breast tumours. Nature. 2000; 406:747-752.

3. Sorlie T, Perou CM, Tibshirani R, Aas T, Geisler S, Johnsen H, Hastie T, Eisen MB, van de Rijn M, Jeffrey SS, Thorsen T, Quist H, Matese JC, et al. Gene expression patterns of breast carcinomas distinguish tumor subclasses with clinical implications. Proc Natl Acad Sci U S A. 2001; 98:10869-10874.

4. Michor F, Polyak K. The origins and implications of intratumor heterogeneity. Cancer Prevention Research. 2010; 3:1361-1364.

5. Nowell PC. The clonal evolution of tumor cell populations. Science. 1976; 194:23-28.

6. Vogelstein B, Fearon ER, Hamilton SR, Kern SE, Preisinger AC, Leppert M, Nakamura Y, White R, Smits AM, Bos JL. Genetic Alterations during ColorectalTumor Development. N Engl J Med. 1988; 319:525-532.

7. Burrell RA, McGranahan N, Bartek J, Swanton C. The causes and consequences of genetic heterogeneity in cancer evolution. Nature. 2013; 501:338-345.

8. Suzuki Y, Ng SB, Chua C, Leow WQ, Chng J, Liu SY, Ramnarayanan K, Gan A, Ho DL, Ten R, Su Y, Lezhava A, Lai JH, et al. Multiregion ultra-deep sequencing reveals early intermixing and variable levels of intratumoral heterogeneity in colorectal cancer. Mol Oncol. 2017; 11:124-139.

9. Dexter DL, Kowalski HM, Blazar BA, Fligiel Z, Vogel R, Heppner GH. Heterogeneity of tumor cells from a single mouse mammary tumor. Cancer Res. 1978; 38:3174-3181.

10. Nik-Zainal S, Van Loo P, Wedge DC, Alexandrov LB, Greenman CD, Lau KW, Raine K, Jones D, Marshall J, Ramakrishna M, Shlien A, Cooke SL, Hinton J, et al. The life history of 21 breast cancers. Cell. 2012; 149:994-1007.

11. McGranahan N, Swanton C. Biological and therapeutic impact of intratumor heterogeneity in cancer evolution. Cancer Cell. 2015; 27:15-26.
12. Gerlinger $\mathrm{M}$, Rowan AJ, Horswell S, Larkin J, Endesfelder D, Gronroos E, Martinez P, Matthews N, Stewart A, Tarpey P, Varela I, Phillimore B, Begum S, et al. Intratumor heterogeneity and branched evolution revealed by multiregion sequencing. N Engl J Med. 2012; 366:883-892.

13. Kuipers J, Jahn K, Beerenwinkel N. Advances in understanding tumour evolution through singlecell sequencing. Biochim Biophys Acta. 2017; 1867:127-138.

14. Williams MJ, Werner B, Barnes CP, Graham TA, Sottoriva A. Identification of neutral tumor evolution across cancer types. Nat Genet. 2016; 48:238-244.

15. Kandoth C, Mclellan MD, Vandin F, Ye K, Niu B, Lu C, Xie M, Zhang Q, Mcmichael JF, Wyczalkowski MA, Leiserson MDM, Miller CA, Welch JS, et al. Mutational landscape and significance across 12 major cancer types. Nature. 2013; 502:333-339.

16. Miller CA, White BS, Dees ND, Griffith M, Welch JS, Griffith OL, Vij R, Tomasson MH, Graubert TA, Walter MJ, Ellis MJ, Schierding W, DiPersio JF, et al. SciClone: inferring clonal architecture and tracking the spatial and temporal patterns of tumor evolution. PLoS Comput Biol. 2014; 10:e1003665.

17. Roth A, Khattra J, Yap D, Wan A, Laks E, Biele J, Ha G, Aparicio S, Bouchard-Côté A, Shah SP. PyClone: statistical inference of clonal population structure in cancer. Nat Methods. 2014; 11:396-398.

18. Hoadley KA, Siegel MB, Kanchi KL, Miller CA, Ding L, Zhao W, He X, Parker JS, Wendl MC, Fulton RS, Demeter RT, Wilson RK, Carey LA, et al. Tumor Evolution in Two Patients with Basal-like Breast Cancer: A Retrospective Genomics Study of Multiple Metastases. PLoS Med. 2016; 13:e1002174.

19. Andor N, Graham TA, Jansen M, Xia LC, Aktipis CA, Petritsch C, Ji HP, Maley CC. Pan-cancer analysis of the extent and consequences of intratumor heterogeneity. Nat Med. 2015; 22:105-113.

20. McGranahan N, Swanton C. Clonal Heterogeneity and Tumor Evolution: Past, Present, and the Future. Cell. 2017; 168:613-628.

21. Weinstein JN, Collisson EA, Mills GB, Shaw KR, Ozenberger BA, Ellrott K, Shmulevich I, Sander C, Stuart JM; Cancer Genome Atlas Research Network. The Cancer Genome Atlas Pan-Cancer analysis project. Nat Genet. 2013; 45:1113-1120.

22. Hudson TJ, Anderson W, Artez A, Barker AD, Bell C, Bernabé RR, Bhan MK, Calvo F, Eerola I, Gerhard DS, Guttmacher A, Guyer M, Hemsley FM, et al; International Cancer Genome Consortium. International network of cancer genome projects. Nature. 2010; 464:993-998.

23. Cancer Genome Atlas Network. Comprehensive molecular portraits of human breast tumours. Nature. 2012; 487:61-70.

24. McFarland CD, Korolev KS, Kryukov GV, Sunyaev SR, Mirny LA. Impact of deleterious passenger mutations on cancer progression. Proc Natl Acad Sci U S A. 2013; 110:2910-2915. 
25. Aran D, Sirota M, Butte AJ. Systematic pan-cancer analysis of tumour purity. Nat Commun. 2015; 6:8971.

26. Benjamini Y, Hochberg Y. Controlling the false discovery rate: a practical and powerful approach to multiple testing. J R Stat Soc Ser B. 1995; 57:289-300.

27. Mroz EA, Rocco JW. MATH, a novel measure of intratumor genetic heterogeneity, is high in poor-outcome classes of head and neck squamous cell carcinoma. Oral Oncol. 2013; 49:211-215.

28. Rajput A, Bocklage T, Greenbaum A, Lee JH, Ness SA. Mutant-Allele Tumor Heterogeneity Scores Correlate With Risk of Metastases in Colon Cancer. Clin Colorectal Cancer. 2017; 16:e165-170.

29. Adzhubei IA, Schmidt S, Peshkin L, Ramensky VE, Gerasimova A, Bork P, Kondrashov AS, Sunyaev SR. A method and server for predicting damaging missense mutations. Nat Methods. 2010; 7:248-249.

30. Vogel BE, Muriel JM, Dong C, Xu X. Hemicentins: what have we learned from worms? Cell Res. 2006; 16:872-878.

31. Schultz DW, Klein ML, Humpert AJ, Luzier CW, Persun V, Schain M, Mahan A, Runckel C, Cassera M, Vittal V, Doyle TM, Martin TM, Weleber RG, et al. Analysis of the ARMD1 locus: Evidence that a mutation in HEMICENTIN-1 is associated with age-related macular degeneration in a large family. Hum Mol Genet. 2003; 12:3315-3323.

32. Li M, Liu F, Zhang Y, Wu X, Wu W, Wang XA, Zhao S, Liu S, Liang H, Zhang F, Ma Q, Xiang S, Li H, et al. Wholegenome sequencing reveals the mutational landscape of metastatic small-cell gallbladder neuroendocrine carcinoma (GB-SCNEC). Cancer Lett. 2017; 391:20-27.

33. Ledgerwood LG, Kumar D, Eterovic AK, Wick J, Chen K, Zhao H, Tazi L, Manna P, Kerley S, Joshi R, Wang L, Chiosea SI, Garnett JD, et al. The degree of intratumor mutational heterogeneity varies by primary tumor sub-site. Oncotarget. 2016; 7:27185-27198. https://doi.org/10.18632/oncotarget.8448.

34. Sisto M, D'Amore M, Lofrumento DD, Scagliusi P, D'Amore S, Mitolo V, Lisi S. Fibulin-6 expression and anoikis in human salivary gland epithelial cells: Implications in Sjogren's syndrome. Int Immunol. 2009; 21:303-311.

35. Chowdhury A, Herzog C, Hasselbach L, Khouzani HL, Zhang J, Hammerschmidt M, Rudat C, Kispert A, Gaestel M, Menon MB, Tudorache I, Hilfiker-Kleiner D, Mühlfeld C, et al. Expression of fibulin-6 in failing hearts and its role for cardiac fibroblastmigration. Cardiovasc Res. 2014; 103:509-520.

36. Timpl R, Sasaki T, Kostka G, Chu ML. Fibulins: A versatile family of extracellular matrix proteins. Nat Rev Mol Cell Biol. 2003; 4:479-89.

37. Yue W, Sun Q, Landreneau R, Wu C, Siegfried JM, Yu J, Zhang L. Fibulin-5 suppresses lung cancer invasion by inhibiting matrix metalloproteinase-7 expression. Cancer Res. 2009; 69:6339-6346.

38. Thippeswamy H, Paul P, Purushottam M, Philip M, Jain S, Chandra PS. Estrogen pathway related genes and their association with risk of postpartum psychosis: A case control study. Asian J Psychiatr. 2017; 26:82-85.

39. Vogel BE, Hedgecock EM. Hemicentin, a conserved extracellular member of the immunoglobulin superfamily, organizes epithelial and other cell attachments into oriented line-shaped junctions. Development. 2001; 128:883-89.

40. Havugimana PC, Hart GT, Nepusz T, Yang H, Turinsky AL, Li Z, Wang PI, Boutz DR, Fong V, Phanse S, Babu M, Craig SA, Hu P, et al. A census of human soluble protein complexes. Cell. 2012; 150:1068-1081.

41. Godbout R, Packer M, Bie W. Overexpression of a DEAD box protein (DDX1) in neuroblastoma and retinoblastoma cell lines. J Biol Chem. 1998; 273:21161-21168.

42. Bléoo S, Sun X, Hendzel MJ, Rowe JM, Packer M, Godbout R. Association of human DEAD box protein DDX1 with a cleavage stimulation factor involved in $3^{\prime}$-end processing of pre-MRNA. Mol Biol Cell. 2001; 12:3046-3059.

43. Guimbellot JS, Erickson SW, Mehta T, Wen H, Page GP, Sorscher EJ, Hong JS. Correlation of microRNA levels during hypoxia with predicted target mRNAs through genome-wide microarray analysis. BMC Med Genomics. 2009; 2:15.

44. Gilkes DM, Semenza GL. Role of hypoxia-inducible factors in breast cancer metastasis. Future Oncol. 2013; 9:1623-1636.

45. Semenza GL. The hypoxic tumor microenvironment: A driving force for breast cancer progression. Biochim Biophys Acta. 2016; 1863:382-391.

46. Han C, Liu Y, Wan G, Choi HJ, Zhao L, Ivan C, He X, Sood AK, Zhang X, Lu X. The RNA-binding protein DDX1 promotes primary microRNA maturation and inhibits ovarian tumor progression. Cell Rep. 2014; 8:1447-1460.

47. Wykoff CC, Beasley NJP, Watson PH, Turner KJ, Pastorek J, Sibtain A, Wilson GD, Turley H, Talks KL, Maxwell PH, Pugh CW, Ratcliffe PJ, Harris AL. Hypoxiainducible expression of tumor-associated carbonic anhydrases. Cancer Res. 2000; 60:7075-83.

48. Valastyan S, Weinberg RA. Tumor metastasis: Molecular insights and evolving paradigms. Cell. 2011; 147:275-292.

49. Zack TI, Schumacher SE, Carter SL, Cherniack AD, Saksena G, Tabak B, Lawrence MS, Zhang CZ, Wala J, Mermel CH, Sougnez C, Gabriel SB, Hernandez B, et al. Pan-cancer patterns of somatic copy number alteration. Nat Genet. 2013; 45:1134-1140.

50. Shlien A, Malkin D. Copy number variations and cancer. Genome Med. 2009; 1:62.

51. Khurana E, Fu Y, Chakravarty D, Demichelis F, Rubin MA, Gerstein M. Role of non-coding sequence variants in cancer. Nat Rev Genet. 2016; 17:93-108.

52. Scacheri CA, Scacheri PC. Mutations in the non-coding genome. Curr Opin Pediatr. 2016; 27:659-664.

53. Jones PA, Baylin SB. The fundamental role of epigenetic events in cancer. Nat Rev Genet. 2002; 3:415-428.

54. ENCODE Project Consortium. The ENCODE (ENCyclopedia Of DNA Elements) Project. Science. 2004; 306:636-40. 